\title{
Diagnostic accuracy of home vs. ambulatory blood pressure monitoring in untreated and treated hypertension
}

\author{
Efthimia G Nasothimiou, Dimitrios Tzamouranis, Vagia Rarra, Leonidas G Roussias and George S Stergiou
}

Several studies with relatively small size and different design and end points have investigated the diagnostic ability of home blood pressure (HBP). This study investigated the usefulness of HBP compared with ambulatory monitoring (ABP) in diagnosing sustained hypertension, white coat phenomenon (WCP) and masked hypertension (MH) in a large sample of untreated and treated subjects using a blood pressure (BP) measurement protocol according to the current guidelines. A total of 613 subjects attending a hypertension clinic (mean age $53 \pm 12.4$ (s.d.) years, men $57 \%$, untreated $59 \%$ ) had measurements of clinic BP (three visits, triplicate measurements per visit), HBP ( 6 days, duplicate morning and evening measurements) and awake ABP (20-min intervals) within 6 weeks. Sustained hypertension was diagnosed in $50 \%$ of the participants by ABP and HBP (agreement $89 \%, \kappa=0.79$ ), WCP in 14 and $15 \%$, respectively (agreement $89 \%, \kappa=0.56$ ) and MH in $16 \%$ and $15 \%$ (agreement $88 \%, \kappa=0.52)$. Only $4 \%$ of the subjects $(27 / 613)$ showed clinically significant diagnostic disagreement with BP deviation $>5 \mathrm{~mm} \mathrm{Hg}$ above the diagnostic threshold (for HBP or ABP). By taking ABP as reference, the sensitivity, specificity, positive and negative predictive value of HBP in detecting sustained hypertension were 90, 89, 89 and $90 \%$, respectively, WCP 61,94 , 64 and $94 \%$ and $\mathrm{MH} \mathrm{60,93,60}$ and $93 \%$. Similar diagnostic agreement was found in untreated and treated subjects. HBP appears to be a reliable alternative to ABP in the diagnosis of hypertension and the detection of WCP and MH in both untreated and treated subjects.

Hypertension Research (2012) 35, 750-755; doi:10.1038/hr.2012.19; published online 23 February 2012

Keywords: ambulatory blood pressure monitoring; home blood pressure monitoring; masked hypertension; white coat hypertension

\section{INTRODUCTION}

Self-monitoring of blood pressure (BP) by patients at home (HBP) is being widely used in several countries and endorsed by hypertension guidelines in Europe, US and elsewhere. ${ }^{1-4}$ HBP monitoring shares most of the advantages of ambulatory BP monitoring (ABP), despite the fact that the two methods are applied in different daily life conditions. The main clinical needs for applying these methods are the white coat and masked hypertension ( $\mathrm{MH})$ phenomena, which are common in both untreated and treated subjects and are missed when evaluation is exclusively based on clinic BP measurements. ${ }^{1-4}$

Early research on ABP monitoring demonstrated its diagnostic and prognostic ability and established its role in the management of hypertension. ${ }^{1}$ Indeed, the existence and clinical relevance of the white coat and the $\mathrm{MH}$ phenomena has been defined using $\mathrm{ABP}$ monitoring. ${ }^{1,5}$ Despite the wide use of HBP monitoring in the community, only recently has evidence accumulated on the diagnostic $^{6}$ and prognostic ${ }^{7}$ usefulness of the method. On the basis of this evidence, recent European and American guidelines on HBP monitoring adopted an entirely different position and recommended for the first time that HBP should be offered to almost all subjects with elevated $\mathrm{BP}^{2,3}$ This recommendation appears to be stronger for the long-term follow-up of treated hypertensives, ${ }^{8}$ in whom there is additional benefit due to the improvement of hypertension control rates when applying HBP monitoring. ${ }^{9}$ On the other hand, the evidence on the usefulness of HBP in the initial evaluation and diagnosis is less clear, and it has been argued that ABP is more appropriate for this indication. ${ }^{2}$

Several studies have assessed the diagnostic ability of HBP by taking $\mathrm{ABP}$ as reference. ${ }^{6}$ However, they had relatively small sample size, did not always apply HBP monitoring according to guidelines and did not examine all the potential diagnoses together (sustained, white coat and $\mathrm{MH}$ ) and in both untreated and treated subjects. $^{6}$

The aim of this study was to investigate the ability of HBP to diagnose sustained, white coat and $\mathrm{MH}$ by taking $\mathrm{ABP}$ as the reference in a large sample of untreated and treated subjects referred to a BP clinic and using a standardized protocol for all the measurement methods according to the current guidelines. 


\section{METHODS}

\section{Study design}

A data set of subjects assessed with clinic BP, ABP and HBP measurements was constructed using data from previous prospective studies conducted in a university hospital BP clinic from 1994 to 2007. Participants were consecutive adults referred for elevated $\mathrm{BP}$, untreated or on stable antihypertensive treatment for $\geqslant 4$ weeks. They were invited to participate in trials involving measurements of clinic BP, HBP and ABP using a standard protocol as described below. Exclusion criteria were severe renal, cardiac or other systemic diseases, sustained arrhythmia and evidence of secondary hypertension. The protocol of the present study was approved by the hospital scientific committee, which did not require an informed consent for this analysis of the patients' BP data and the retrospective collection of other data from patients' records.

\section{BP measurements}

In each participant, clinic BP, ABP and HBP measurements were performed within 6 weeks. After a pre-study visit, clinic BP was measured in three study visits, before, between and after HBP and ABP monitoring. Triplicate BP measurements were taken at each clinic visit after $5 \mathrm{~min}$ sitting rest and with at least 1 min between recordings using a standard mercury sphygmomanometer (bladder $12 \times 23 \mathrm{~cm}$ or $15 \times 35 \mathrm{~cm}$ where appropriate, Korotkoff phase $\mathrm{V}$ for diastolic BP). Measurements were taken by physicians who fulfilled the British Hypertension Society Protocol criteria for observer agreement in BP measurement. ${ }^{10}$

ABP was monitored using validated oscillometric devices SpaceLabs 90207 or 90217 (SpaceLabs Inc., Redmond, WA, USA, bladder size $12 \times 23 \mathrm{~cm}$, or $14 \times 30 \mathrm{~cm}$ where appropriate). ${ }^{11,12}$ The devices were programmed to measure BP at 20-min intervals for $24 \mathrm{~h}$ and were applied on a routine working day. Subjects were instructed to follow their usual daily activities but to avoid extreme physical activities and to remain still with the forearm extended during each BP measurement. A brief diary was provided to report the time when they went to bed and arose.

HBP was monitored on six routine working days within 2 weeks ${ }^{13}$ using validated oscillometric arm devices Omron HEM-705CP (Omron Healthcare Europe BV, Hoofddorp, The Netherlands; inflatable bladder size $12 \times 23 \mathrm{~cm}$ or $14 \times 28 \mathrm{~cm}$ according to the individual's arm circumference), Omron IC (Omron Healthcare Europe BV; bladder $12 \times 23 \mathrm{~cm}$ and $14 \times 28 \mathrm{~cm}$ ), or Omron 705IT (Omron Healthcare Europe BV; bladder $13 \times 23 \mathrm{~cm}$ or $15 \times 30 \mathrm{~cm}$ ). ${ }^{14}$ Participants were trained in the conditions of HBP measurement and the use of the devices. They were instructed to take duplicate morning (0600-1000 hours, before drug intake if treated) and evening (1800-2200 hours) measurements after 5 min sitting rest and with 1-min interval between measurements. A form was supplied to the participants to report all their HBP readings, which were also printed by the device memory (Omron HEM-705CP) or downloaded through a computer link (Omron 705IT and Omron IC). Before each ABP or HBP monitoring session, the accuracy of the devices was tested in each individual against a standard mercury sphygmomanometer (three successive readings; $\mathrm{Y}$ connector) to ensure that there was no consistent difference of $>10 \mathrm{~mm} \mathrm{Hg}$ in measured BP.

\section{Definitions}

The BP threshold for hypertension diagnostic was $140 \mathrm{~mm} \mathrm{Hg}$ and/or $90 \mathrm{~mm} \mathrm{Hg}$ (systolic/diastolic) for clinic BP and $135 \mathrm{~mm} \mathrm{Hg}$ and/or $85 \mathrm{~mm} \mathrm{Hg}$ for HBP and awake ABP. ${ }^{1}$ Sustained hypertension was defined as elevated clinic and out-of-clinic BP (home or awake ambulatory). White coat phenomenon (WCP) was defined as elevated clinic and low out-of-clinic BP in both treated and untreated subjects, and $\mathrm{MH}$ the reverse (low clinic and elevated out-of-clinic BP).

\section{Analysis}

Participants who provided fewer than eight valid HBP readings were excluded. HBP readings that were $>50 \%$ higher than the next highest HBP reading of the individual subject were considered erroneous and were discarded, as were measurements with systolic BP $<60 \mathrm{~mm} \mathrm{Hg}$ or $>250 \mathrm{~mm} \mathrm{Hg}$ and those with diastolic $\mathrm{BP}<30 \mathrm{~mm} \mathrm{Hg}$ or $>150 \mathrm{~mm} \mathrm{Hg}$. All HBP readings were averaged to give a single number per individual.
ABP data and additional recorded information from the report files generated by the ambulatory monitors were batch imported and organized in a relational database (Microsoft Access Office Premium 2000, Ireland) using a Visual Basic program (Microsoft Visual Basic 6 Professional Edition, Ireland 2000). This program designed by LGR (author) for statistical analysis of ABPderived data reads the ASCII text files generated by the ABP monitor and performs multiple data procedures and analyses, including flagging erroneous readings, valid readings and duplicate readings (repeats), and calculates the average awake and asleep ABP, according to each individual's report for in-bed and out-of-bed period. ABP recordings with $<20$ valid awake readings were excluded. Early readings taken $<20 \mathrm{~min}$ after the monitor had been attached to subjects were also excluded because these had been taken in the clinic. The average of the second and third clinic BP reading of the three study visits (six readings) was calculated to give clinic $\mathrm{BP}$ per individual.

Student's paired $t$-test was used for the comparison of BP measurements in the same subjects with Bonferroni's correction for multiple comparisons applied where appropriate. We estimated that using a $5-\mathrm{mm} \mathrm{Hg}$ cutoff for clinical significant difference between systolic awake ABP and HBP, the power of a 613 subjects study sample (retrospective data set analysis) was up to $100 \%$ ( $\alpha=0.05$, two-tailed), indicating an oversized study. Student's unpaired $t$-test was used to compare values among subgroup of participants. The diagnostic ability of HBP (sensitivity, specificity, positive and negative predictive value) to detect sustained, WCP and MH in the total population and separately in treated and untreated subgroups was investigated.

The $\kappa$ statistic was used to determine the level of agreement in diagnoses made by ABP and HBP. Multivariable linear regression models were applied to investigate independent predictors of the ABP-HBP difference. Binary logistic regression models were used to identify independent factors associated with WCP and MH diagnosed by HBP or ABP monitoring. Covariates included in the models were age, gender, body mass index, systolic/diastolic clinic BP, sex, number of HBP and awake ABP readings, cardiovascular disease, diabetes, smoking, alcohol intake and treatment for hypertension. Current smoking was defined as daily smoking or smoking cessation for $<1$ year and alcohol intake as consumption of any alcohol amount per week. A probability value $P<0.05$ was considered statistically significant. Analysis was performed using the PASW 18 (SPSS release 18.0; SPSS Inc., Chicago, IL, USA).

\section{RESULTS}

\section{Patients' characteristics}

A total of 710 cases were reviewed for inclusion in the analysis and 97 (13.7\%) were excluded. Forty-eight cases were excluded due to inadequate $\mathrm{HBP}$ and/or ABP readings, 34 because evaluation has been performed more than once (first assessment was used), five due to treatment change during the study, five due to acute disease during the study and five due to other reasons. A total of 613 subjects were finally included in the analysis. Participants' characteristics are presented in Table 1, for the total study population and separately for untreated and treated subjects.

\section{BP measurements}

The average number of valid clinic BP readings included in the analysis was $5.0 \pm 1.2$ (range 2-6), HBP $21.3 \pm 4.2$ (range 8-24) and awake ABP $42.3 \pm 6.7$ (range 21-60). In the total population, clinic BP was higher than HBP (mean difference, $3.4 \pm 12.9 / 4.6 \pm 7.6 \mathrm{~mm} \mathrm{Hg}$, systolic/diastolic, $P<0.001$ for both) and awake ABP (mean difference, $3.1 \pm 14 / 2.8 \pm 8.4 \mathrm{~mm} \mathrm{Hg}$, systolic/diastolic, for both $P<0.001$ ), whereas there is no difference between systolic HBP and awake ABP, yet diastolic HBP was lower by $1.8 \pm 7.1 \mathrm{~mm} \mathrm{Hg}(P<0.001)$ (Table 2). In treated subjects, systolic HBP was slightly higher than awake ABP, whereas the reverse was true in the untreated subjects (Table 2).

Significant associations were found between HBP and awake ABP ( $r=0.63 / 0.76$, systolic/diastolic, $P<0.001$ for both), clinic BP and HBP $(r=0.62 / 0.73, P<0.001$ for both $)$ and clinic BP and awake ABP $(r=0.53 / 0.69, P<0.001$ for both). 
Table 1 Demographics of the total study population and of untreated and treated subjects

\begin{tabular}{|c|c|c|c|c|}
\hline & Total, $\mathrm{N}=613$ & Untreated, $\mathrm{N}=361(59 \%)$ & Treated, $\mathrm{N}=252$ (41\%) & P-value a \\
\hline Age (years) & $53 \pm 12$ & $49 \pm 12$ & $58 \pm 11$ & $<0.001$ \\
\hline Current smoking (yes/no, \%) & 22 & 26 & 16 & NS \\
\hline Alcohol intake (yes/no, \%)c & 39 & 39 & 39 & NS \\
\hline Cardiovascular disease (\%) & 5.7 & 2.4 & 10.6 & $<0.001$ \\
\hline
\end{tabular}

aFor comparison between untreated and treated subjects.

bDaily smoking or smoking cessation for $<1$ year.

${ }^{c}$ Consumption of any alcohol amount per week.

Table 2 Blood pressure in the total study population and in untreated and treated subjects

\begin{tabular}{lccr}
\hline Blood pressure $(\mathrm{mm} \mathrm{Hg})$ & Total, N=613 & Treated, N=252 (41\%) & Untreated, N=361 (59\%) \\
\hline Clinic SBP & $140 \pm 15$ & $135 \pm 15$ & $143 \pm 14$ \\
Clinic DBP & $89 \pm 11$ & $84 \pm 10$ & $94 \pm 9.2$ \\
Home SBP & $136 \pm 14^{* *}$ & $134 \pm 14$ & $138 \pm 14^{* *}$ \\
Home DBP & $85 \pm 10^{* *}$ & $80 \pm 8^{* *}$ & $88 \pm 9^{* *}$ \\
Awake ambulatory SBP & $137 \pm 14^{* *}$ & $132 \pm 13^{*}$ & $140 \pm 13^{* *+}$ \\
Awake ambulatory DBP & $87 \pm 11^{* *++}$ & $81 \pm 10^{* *+}$ & $<0.001$ \\
\hline
\end{tabular}

Abbreviations: BP, blood pressure; DBP, diastolic blood pressure; SBP, systolic blood pressure.

aFor comparison between untreated and treated subjects.

${ }^{*} P<0.001,{ }^{*} P<0.01$, for comparison with clinic BP; ${ }^{++} P<0.001,{ }^{+} P<0.05$, for comparison with home BP.

\section{Diagnostic evaluation}

In Figure 1, participants were classified according to the diagnosis of normotension (clinic and out-of-clinic), sustained hypertension (clinic and out-of-clinic), white coat and MH phenomena by HBP and awake ABP method, respectively, for the total population (1a) and in treated (1b) and untreated subjects (1c).

Sustained hypertension was diagnosed in $50 \%$ by either ABP or HBP (diagnostic agreement $89 \%, \kappa=0.79$ ), WCP in 14 and $15 \%$, respectively (agreement $89 \%, \kappa=0.56$ ) and $\mathrm{MH}$ in 16 and $15 \%$ (agreement $88 \% ; \kappa=0.52$ ) (Figure 1; Table 3). $\mathrm{MH}$ tended to be more common in treated ( $23 \%$ by HBP; $20 \%$ by ABP) compared with untreated subjects ( $10 \%$ by HBP; $12 \%$ by ABP, $P<0.05 / \mathrm{NS}$ compared with treated), and this was also the case for WCP in treated ( $16 \%$ by HBP; $17 \%$ by $\mathrm{ABP}$ ) vs. untreated subjects ( $12 \%$ by $\mathrm{HBP}, 13 \%$ by $\mathrm{ABP}$, $P=$ NS compared with treated). One-third of the treated and $10 \%$ of untreated subjects had controlled clinic and out-of-clinic BP.

By taking $\mathrm{ABP}$ as the reference method, the sensitivity, specificity, positive and negative predictive value of HBP in detecting sustained, WCP and MH are presented in Table 3 for the total study population and separately in untreated and treated subjects.

Predictors of the diagnostic disagreement between $\mathrm{HBP}$ and ABP Among the total of 613 cases, 65 (11\%) had disagreement between $\mathrm{HBP}$ and $\mathrm{ABP}$ in the diagnosis of sustained hypertension. In 27 of these 65 cases $(42 \%)$, the BP difference away from the corresponding diagnostic threshold was within $5 \mathrm{~mm} \mathrm{Hg}$, which was considered as clinically uncertain discrepancy. Disagreement between HBP and ABP in the diagnosis of WCP was found in 65 subjects (11\%) and in the diagnosis of $\mathrm{MH}$ in $76(12 \%)$. This diagnostic discrepancy in WCP was considered as clinically uncertain (BP within $5 \mathrm{~mm} \mathrm{Hg}$ from diagnostic threshold) in 27 subjects (42\%) and in $\mathrm{MH}$ in 48 (63\%).
Independent predictors of the difference between HBP and awake ABP awake derived from multivariable regression models are presented in Table 4.

\section{Predictors of white coat and MH phenomena detected by HBP or $\mathrm{ABP}$}

The same potential predictors of white coat and $\mathrm{MH}$ phenomena were entered in binary regression models separately for HBP and ABP. The independent predictors of white coat and $\mathrm{MH}$ detected by HBP or ABP are presented in Table 5.

\section{DISCUSSION}

The main finding of this study is the consistent and substantial agreement between HBP and ABP in the diagnosis of sustained hypertension, WCP and MH in both untreated and treated subjects. HBP appeared to have high sensitivity and specificity when tested by taking ABP monitoring as reference in the diagnosis of sustained hypertension. Lower but still satisfactory was the sensitivity of HBP in the diagnosis of white coat and $\mathrm{MH}$ phenomenon whereas the specificity was $>90 \%$.

Several previous studies have investigated the diagnostic ability of HBP by taking ABP as reference. ${ }^{6}$ Most of these studies have looked at selected diagnostic phenotypes of hypertension (sustained, white coat, masked or resistant) and each one included 44-276 subjects, treated or untreated or both. ${ }^{6}$ The strengths of the present study are the large sample size $(n=613)$, the homogeneity of the data derived from a single BP clinic, the standardized BP measurement protocol according to the current guidelines, ${ }^{1,2}$ the investigation of all the diagnostic scenarios in hypertension and the inclusion of usual population with elevated BP, which allows the generalization of the findings in the vast majority of the hypertensive population. Furthermore, the diagnostic 
value of HBP was explored separately in untreated and treated subjects, providing thereby valuable information on the usefulness of the method in the initial evaluation and the long-term follow-up.

In this study, there was a striking similarity in the proportion of subjects with normotension, sustained, white coat and $\mathrm{MH}$ diagnosed

a

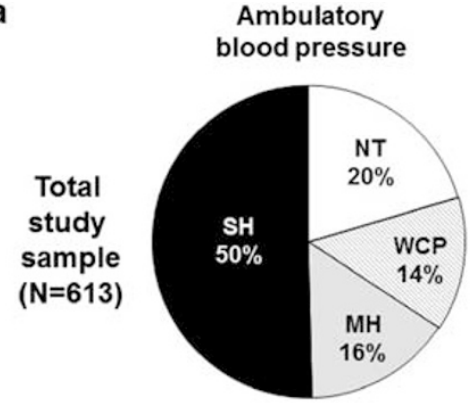

b

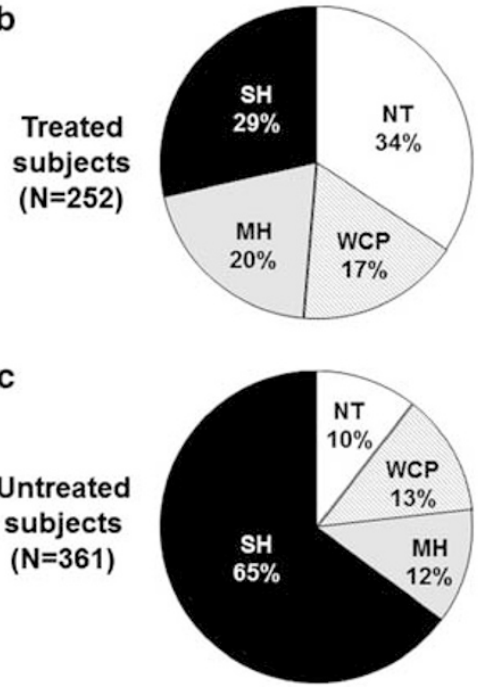

Figure 1 Subjects classified as having normotension (NT), sustained hypertension (SH), white coat phenomenon (WCP) or masked hypertension phenomenon $(\mathrm{MH})$ on the basis of clinic and awake ambulatory or home blood pressure measurements. by $\mathrm{ABP}$ or $\mathrm{HBP}$. However, similar proportions do not necessarily mean that the same subjects are detected by the two methods. A recent systematic review on the diagnostic value of HBP presented 16 studies (two of them in children and adolescents) providing data on sensitivity, specificity, positive and negative predictive value by taking $A B P$ as reference. ${ }^{6}$ In untreated subjects, the sensitivity for hypertension diagnosis varied between $48-100 \%$ and the specificity between $44-93 \%$ and in treated $52-97$ and $63-84 \%$ respectively, which give a trend but is rather inconclusive. ${ }^{6}$ Diagnostic agreement between the two methods expressed by $\kappa$ statistic ranged from 38 to 59, which reflect fair-to-moderate agreement.

In the present study, there was a consistent trend towards high specificity and negative predictive value with tight confidence intervals, and less so for sensitivity and positive predictive value. Moreover, HBP showed substantial agreement with ABP in the diagnosis of hypertension, which is superior to that of previous studies. ${ }^{6}$ This appears to be attributed to strictly standardized measurement condi-

Table 4 Factors associated with the difference between awake ambulatory and home blood pressure in the total study population $(n=613)$

\begin{tabular}{lcl}
\hline Variables & B coefficient & P-value \\
\hline Systolic BP difference & & \\
Age (years) & -0.17 & $<0.001$ \\
Clinic systolic BP (mm Hg) & -0.22 & $<0.001$ \\
Clinic diastolic BP (mm Hg) & 0.28 & $<0.001$ \\
Gender (1, male; 2, female) & 2.54 & $<0.05$ \\
Number of home BP readings & 0.33 & $<0.01$ \\
Current smoker (0, no; 1, yes) & -2.09 & $<0.05$ \\
& & \\
Diastolic BP difference & & $<0.001$ \\
Age (years) & -0.17 & $<0.001$ \\
Clinic systolic BP (mm Hg) & -0.22 & $<0.001$ \\
Clinic diastolic BP (mm Hg) & 0.28 & $<0.05$ \\
Gender (1, male; 2, female) & 2.54 & $<0.01$ \\
Number of home BP readings & 0.33 & $<0.05$ \\
Current smoker (0, no; 1, yes) & -2.09 &
\end{tabular}

Abbreviation: BP, blood pressure.

Covariates tested in the multivariable regression model: age, gender, body mass index, systolic/ diastolic clinic BP, number of home and awake ambulatory BP readings, cardiovascular disease, diabetes, smoking, alcohol intake and treatment for hypertension.

Table 3 Diagnostic accuracy of home blood pressure taking ambulatory blood pressure as reference method in the total study population $(n=613)$ and in untreated $(n=361)$ and treated subjects $(n=252)$

\begin{tabular}{|c|c|c|c|c|c|c|c|}
\hline Diagnosis & $\begin{array}{c}\text { Study } \\
\text { population }\end{array}$ & $\begin{array}{c}\text { Sensitivity } \\
\text { (\%) }\end{array}$ & $\begin{array}{l}\text { Specificity } \\
(\%)\end{array}$ & $\begin{array}{c}\text { Positive predictive } \\
\text { value (\%) }\end{array}$ & $\begin{array}{c}\text { Negative predictive } \\
\text { value (\%) }\end{array}$ & $\begin{array}{c}\text { Agreement } \\
\text { (\%) }\end{array}$ & $\kappa$ Statistic \\
\hline \multirow[t]{3}{*}{ Sustained hypertension } & Total & $90(86-93)$ & $89(85-92)$ & $89(85-92)$ & $90(86-93)$ & 89 & $0.79 *$ \\
\hline & Treated & $86(76-93)$ & 94 (89-97) & $85(74-92)$ & $94(90-97)$ & 92 & $0.80 *$ \\
\hline & Untreated & $91(86-94)$ & $82(74-88)$ & $90(86-94)$ & $83(75-89)$ & 88 & $0.73^{*}$ \\
\hline \multirow[t]{3}{*}{ White coat phenomenon } & Total & $61(50-71)$ & $94(92-96)$ & $64(52-74)$ & $94(91-96)$ & 89 & $0.56^{*}$ \\
\hline & Treated & $74(58-86)$ & $95(91-98)$ & $76(60-87)$ & 95 (91-97) & 92 & $0.69 *$ \\
\hline & Untreated & $50(35-65)$ & $93(90-96)$ & $52(37-67)$ & $93(89-95)$ & 88 & $0.44^{*}$ \\
\hline \multirow[t]{3}{*}{ Masked hypertension phenomenon } & Total & $60(49-69)$ & $93(90-95)$ & 60 (49-69) & $93(90-95)$ & 88 & $0.52^{*}$ \\
\hline & Treated & $53(39-67)$ & 85 (79-90) & $47(34-61)$ & 88 (82-92) & 79 & $0.69 *$ \\
\hline & Untreated & $67(51-81)$ & 98 (95-99) & 78 (61-90) & 96 (93-98) & 94 & $0.40 *$ \\
\hline
\end{tabular}

${ }^{*} P<0.001: 95 \% \mathrm{Cl}$ in parentheses. 
Table 5 Predictors of white coat and masked hypertension phenomena detected by home or ambulatory blood pressure in the total study population $(n=613)$

\begin{tabular}{|c|c|c|c|c|}
\hline \multirow[b]{2}{*}{ Variables } & \multicolumn{2}{|c|}{ Home $B P$} & \multicolumn{2}{|c|}{ Ambulatory BP } \\
\hline & Odds ratio & P-value & Odds ratio & P-value \\
\hline \multicolumn{5}{|c|}{ White coat hypertension phenomenon } \\
\hline Clinic systolic BP (mm Hg) & 0.99 & n.s. & 1.02 & $<0.05$ \\
\hline Gender ( 1 , male; 2 , female) & 2.45 & $<0.001$ & 3.38 & $<0.001$ \\
\hline Current smoker ( 0, no; 1 , yes) & 0.42 & $<0.01$ & 0.4 & $<0.01$ \\
\hline \multicolumn{5}{|l|}{ Masked hypertension phenomenon } \\
\hline Clinic systolic BP $(\mathrm{mm} \mathrm{Hg})$ & 0.95 & $<0.05$ & 0.95 & $<0.01$ \\
\hline Clinic diastolic BP (mm Hg) & 0.92 & $<0.05$ & 0.95 & $<0.01$ \\
\hline Number of home BP readings & 1.0 & n.s. & 1.1 & $<0.05$ \\
\hline Current smoker ( 0 , no; 1 , yes) & 2.4 & $<0.01$ & 1.5 & n.s. \\
\hline
\end{tabular}

Abbreviations: BP, blood pressure; n.s., non-significant.

Covariates tested in the multivariable binary regression model: age, gender, body mass index, systolic/diastolic clinic BP, number of home and awake ambulatory BP readings, cardiovascular disease, diabetes, smoking, alcohol intake and treatment for hypertension.

tions and protocol according to the current guidelines applied in a single research setting and in a large study sample. In the total study population only $4 \%$ of subjects showed clinically significant diagnostic disagreement with $\mathrm{BP}$ deviation $>5 \mathrm{~mm} \mathrm{Hg}$ above the diagnostic threshold (for HBP or ABP). For the rest of the cases with disagreement, the BP deviation was within $5 \mathrm{~mm} \mathrm{Hg}$ above the threshold, a difference which, due to the known BP variability, should be considered by the practicing physician as uncertain to decide treatment titration.

The investigation for potential predictors of the ABP-HBP difference showed the level of systolic BP to be inversely related with the difference, suggesting that for raised BP levels ABP and HBP tend to provide similar results. On the other hand, in lower BP, values close the diagnostic threshold disagreement is inevitably increased, since a few $\mathrm{mm} \mathrm{Hg}$ difference attributed to BP variability might lead to disagreement, which is more often arithmetic (within $5 \mathrm{~mm} \mathrm{Hg}$ ) rather than clinically important. A significant influence of age on the ABP-HBP difference was also shown, with elderly patients showing smaller difference than the younger ones. This finding is in line with a recent meta-analysis by Ishikawa et al., ${ }^{15}$ which showed no difference between awake ABP and HBP in older subjects, whereas in the younger HBP was lower.

The Pamela general population study provided a direct ABP/ HBP comparison in 1529 subjects and showed that of patients with white coat hypertension diagnosed by $\mathrm{ABP} 70 \%$ were detected by HBP, yet only two HBP readings were obtained in that study. ${ }^{16}$ Smaller studies, showed diagnostic disagreement of the two methods in the detection of WCP within a range of $13-25 \% .{ }^{17-19}$ In the current study, superior agreement between the two methods in diagnosing WCP was found which as mentioned before might be attributed to the standardized measurement methods according to guidelines, which fully exhausted their potential. The sensitivity of HBP in detecting WCP was superior in the treated subgroup than the untreated and inferior to specificity values. This trend was identified in some other smaller studies, ${ }^{17,18,20}$ with sensitivity settled around $50 \%$ and specificity around $80 \%$.

Regarding the $\mathrm{MH}$ phenomenon, in the Pamela study, ${ }^{16} \mathrm{HBP}$ confirmed only $57 \% / 45 \%$ (systolic/diastolic) of patients having $\mathrm{MH}$ by ABP. A cross-sectional study of 438 subjects attending a hypertension clinic also found similar proportions of masked hypertensives diagnosed by ABP or HBP (14.2 and 11.9\%, respectively), yet among subjects with $\mathrm{MH}$, there was disagreement in the diagnosis between the two methods in $23 \%$ for systolic and $30 \%$ for diastolic BP. ${ }^{21}$ In this study, in untreated hypertensives, the diagnostic agreement exceeded $90 \%$, whereas in treated ones was about $80 \%$. As for WCP, the sensitivity of HBP was lower than specificity in the detection of $\mathrm{MH}$, yet appeared to be satisfactory for clinical practice.

The determinants of white coat and $\mathrm{MH}$ phenomena have been intensively investigated in previous studies. In a recent metaanalysis of 27 studies $(n=12127)$, Ishikawa et al. ${ }^{15}$ showed that increasing age, female gender and not using oscillometric device for clinic BP, predispose to WCP detecting by ABP. In a study of 5716 subjects, white coat hypertension detected by $\mathrm{ABP}$ was more common among older adults, females and non-smokers. ${ }^{22}$ These data are in line with the current findings, suggesting that female gender and non-smoking are established determinants of the WCP, irrespective of the measurement method (ABP or HBP). This was also the case with $\mathrm{MH}$, where lower clinic $\mathrm{BP}$ was associated with $\mathrm{MH}$, irrespective of the measurement method, and smoking which is an established predictor of $\mathrm{MH}$ diagnosed by $\mathrm{ABP},{ }^{23}$ was also a predictor of $\mathrm{MH}$ detected by HBP. Taken together, the available evidence suggests that the white coat and $\mathrm{MH}$ phenomena have consistent clinical predictors, irrespective of the method used for their detection (ABP or HBP), which supports the view that the two methods are interchangeable.

In conclusion, HBP showed substantial diagnostic agreement with $\mathrm{ABP}$ in the diagnosis of hypertension. The between-methods discrepancy may be attributable to inherent methodological differences. The imperfect reproducibility of both methods ${ }^{24,25}$ also contributes to diagnostic discrepancy, as well as the fact that most of the BP values are close to the diagnostic thresholds, which might lead to disagreement even in cases with BP difference of a few $\mathrm{mmHg}$ only. The recent evidence from meta-analysis of outcome HBP studies, ${ }^{7}$ together with the consistent evidence on the diagnostic ability of HBP suggest that this method should no longer be regarded as a screening test that requires confirmation by $\mathrm{ABP}$, as has been previously proposed, ${ }^{26}$ but as a reliable alternative to $\mathrm{ABP}$. However, $\mathrm{ABP}$ remains a unique tool in the assessment of nocturnal $\mathrm{BP}$ and the detection of non-dippers, as well as for the evaluation of short-term reading-to-reading BP variability. Preliminary evidence suggests that novel home monitors can also evaluate nocturnal $\mathrm{BP},{ }^{27}$ whereas medium-term day-to-day BP variability assessed by self-home measurements has been shown to predict cardiovascular risk beyond the other risk factors. ${ }^{28}$ Given the wide availability of home monitors, their relatively low cost and the good acceptance by users, HBP monitoring should be applied in almost all subjects with elevated BP for both the initial evaluation and the long-term follow-up. . $^{2,3}$

\section{CONFLICT OF INTEREST}

The authors declare no conflict of interest.

\section{ACKNOWLEDGEMENTS}

The home devices used in the study were provided by G Leousis IS Athens, Greece. 
1 O'Brien E, Asmar R, Beilin L, Imai Y, Mallion JM, Mancia G, Mengden T, Myers M, Padfield P, Palatini P, Parati G, Pickering T, Redon J, Staessen J, Stergiou G, Verdecchia $P$. European Society of Hypertension recommendations for conventional, ambulatory and home blood pressure measurement. J Hypertens 2003; 21: 821-848.

2 Parati G, Stergiou GS, Asmar R, Bilo G, de Leeuw P, Imai Y, Kario K, Lurbe E, Manolis A, Mengden T, O'Brien E, Ohkubo T, Padfield P, Palatini P, Pickering T, Redon J, Revera M, Ruilope LM, Shennan A, Staessen JA, Tisler A, Waeber B, Zanchetti A, Mancia G. European Society of Hypertension guidelines for blood pressure monitoring at home: a summary report of the Second International Consensus Conference on Home Blood Pressure Monitoring. J Hypertens 2008; 26: 1505-1526.

3 Pickering TG, Miller NH, Ogedegbe G, Krakoff LR, Artinian NT, Goff D. Call to action on use and reimbursement for home blood pressure monitoring: a joint scientific statement from the American Heart Association, American Society Of Hypertension, and Preventive Cardiovascular Nurses Association. Hypertension 2008; 52: 10-29.

4 Imai Y, Otsuka K, Kawano Y, Shimada K, Hayashi H, Tochikubo O, Miyakawa M, Fukiyama K. Japanese society of hypertension (JSH) guidelines for self-monitoring of blood pressure at home. Hypertens Res 2003; 26: 771-782.

5 Ohkubo T, Kikuya M, Metoki H, Asayama K, Obara T, Hashimoto J, Totsune K, Hoshi H, Satoh H, Imai Y. Prognosis of 'masked' hypertension and 'white-coat' hypertension detected by 24-h ambulatory blood pressure monitoring 10-year follow-up from the Ohasama study. J Am Coll Cardiol 2005; 46: 508-515.

6 Stergiou GS, Bliziotis IA. Home blood pressure monitoring in the diagnosis and treatment of hypertension: a systematic review. Am J Hypertens 2011; 24: 123-134.

7 Stergiou GS, Siontis KC, loannidis JP. Home blood pressure as a cardiovascular outcome predictor: it's time to take this method seriously. Hypertension 2010; 55: 1301-1303.

8 Yasui D, Asayama K, Ohkubo T, Kikuya M, Kanno A, Hara A, Hirose T, Obara T, Metoki H, Inoue R, Totsune K, Hoshi H, Satoh H, Imai Y. Stroke risk in treated hypertension based on home blood pressure: the Ohasama study. Am J Hypertens 2010; 23: 508-514.

9 Cappuccio FP, Kerry SM, Forbes L, Donald A. Blood pressure control by home monitoring: meta-analysis of randomised trials. Br Med J 2004; 329: 145-151.

10 O'Brien E, Petrie J, Littler W, de Swiet M, Padfield PL, Altman DG, Bland M, Coats A, Atkins $N$. An outline of the revised British Hypertension Society protocol for the evaluation of blood pressure measuring devices. J Hypertens 1993; 11: 677-679.

11 O'Brien E, Mee F, Atkins N, O'Malley K. Accuracy of the Spacelabs 90207 determined by to the British Hypertension Society Protocol. J Hypertens 1991; 9 (Suppl 5): S25-S31.

12 Baumgart P, Kamp J. Accuracy of the Spacelabs Medical 90217 ambulatory blood pressure monitor. Blood Press Monit 1998; 3: 303-307.

13 Stergiou GS, Parati G. The optimal schedule for self-monitoring of blood pressure by patients at home. J Hypertens 2007; 25: 1992-1997.

14 dabls Educational Trust. Devices for blood pressure measurement. http://www. dableducational.org, assessed October 2011.

15 Ishikawa J, Ishikawa Y, Edmondson D, Pickering TG, Schwartz JE. Age and the difference between awake ambulatory blood pressure and office blood pressure: a meta-analysis. Blood Press Monit 2011; 16: 159-167.
16 Sega R, Trocino G, Lanzarotti A, Carugo S, Cesana G, Schiavina R, Valagussa F, Bombelli M, Giannattasio C, Zanchetti A, Mancia G. Alterations of cardiac structure in patients with isolated office, ambulatory, or home hypertension: data from the general population (Pressione Arteriose Monitorate E Loro Associazioni [PAMELA] Study). Circulation 2001; 104: 1385-1392.

17 Stergiou GS, Skeva II, Baibas NM, Kalkana CB, Roussias LG, Mountokalakis TD. Diagnosis of hypertension using home or ambulatory blood pressure monitoring: comparison with the conventional strategy based on repeated clinic blood pressure measurements. J Hypertens 2000; 18: 1745-1751.

18 Stergiou GS, Zourbaki AS, Skeva II, Mountokalakis TD. White coat effect detected using self-monitoring of blood pressure at home: comparison with ambulatory blood pressure. Am J Hypertens 1998; 11: 820-827.

19 Den Hond E, Celis H, Vandenhoven G, O'Brien E, Staessen JA. Determinants of whitecoat syndrome assessed by ambulatory blood pressure or self-measured home blood pressure. Blood Press Monit 2003; 8: 37-40.

20 Bayó J, Cos FX, Roca C, Dalfó A, Martín-Baranera MM, Albert B. Home blood pressure self-monitoring: diagnostic performance in white-coat hypertension. Blood Press Monit 2006; 11: 47-52.

21 Stergiou GS, Salgami EV, Tzamouranis DG, Roussias LG. Masked hypertension assessed by ambulatory blood pressure versus home blood pressure monitoring: is it the same phenomenon? Am J Hypertens 2005; 18: 772-778.

22 Dolan E, Stanton A, Atkins N, Den Hond E, Thijs L, McCormack P, Staessen J, O'Brien E. Determinants of white-coat hypertension. Blood Press Monit 2004; 9: 307-309.

23 Hänninen MR, Niiranen TJ, Puukka PJ, Mattila AK, Jula AM. Determinants of masked hypertension in the general population: the Finn-Home study. J Hypertens 2011; 29: 1880-1888.

24 James GD, Pickering TG, Yee LS, Harshfield GA, Riva S, Laragh JH. The reproducibility of average ambulatory, home, and clinic pressures. Hypertension 1988; 11: 545-549.

25 Stergiou GS, Baibas NM, Gantzarou AP, Skeva II, Kalkana CB, Roussias LG, Mountokalakis TD. Reproducibility of home, ambulatory, and clinic blood pressure: implications for the design of trials for the assessment of antihypertensive drug efficacy. Am J Hypertens 2002; 15: 101-104.

26 Pickering TG, Hall JE, Appel LJ, Falkner BE, Graves J, Hill MN, Jones DW, Kurtz T, Sheps SG, Roccella EJ. Recommendations for blood pressure measurement in humans and experimental animals: Part 1: blood pressure measurement in humans: a statement for professionals from the Subcommittee of Professional and Public Education of the American Heart Association Council on High Blood Pressure Research. Hypertension 2005; 45: 142-161.

27 Chonan K, Kikuya M, Araki T, Fujiwara T, Suzuki M, Michimata M, Hashimoto J, Ohkubo T, Hozawa A, Yamamoto N, Miyawaki Y, Matsubara M, Imai Y. Device for the self-measurement of blood pressure that can monitor blood pressure during sleep. Blood Press Monit 2001; 6: 203-205.

28 Kikuya M, Ohkubo T, Metoki H, Asayama K, Hara A, Obara T, Inoue R, Hoshi H, Hashimoto J, Totsune K, Satoh H, Imai Y. Day-by-day variability of blood pressure and heart rate at home as a novel predictor of prognosis: the Ohasama study. Hypertension 2008; 52: 1045-1050. 